\title{
A New Forged Handwriting Detection Method based on Fourier Spectral Density and Variation
}

\author{
${ }^{1}$ Sayani Kundu, ${ }^{2}$ Palaiahnakote Shivakumara, ${ }^{2}$ Anaica Grouver, ${ }^{1}$ Umapada Pal, ${ }^{3}$ Tong \\ Lu and ${ }^{4}$ Michael Blumenstein \\ ${ }^{1}$ Computer Vision and Pattern Recognition Unit, Indian Statistical Institute, Kolkata, India. \\ Email: sayani.frndz@gmail.com,umapada@isical.ac.in. \\ ${ }^{2}$ Faculty of Computer Science and Information Technology, University of Malaya, Kuala Lum- \\ pur, Malaysia. Email: shiva@um.edu.my, anaicagrouver@gmail.com \\ ${ }^{3}$ National Key Lab for Novel Software Technology, Nanjing University, Nanjing, China. \\ Email: lutong@nju.edu.cn \\ ${ }^{4}$ Faculty of Engineering and Information Technology, University of Technology, Sydney, Aus- \\ tralia. Email: Michael.Blumenstein@uts.edu.au.
}

\begin{abstract}
Use of handwriting words for person identification in contrast to biometric features is gaining importance in the field of forensic applications. As a result, forging handwriting is a part of crime applications and hence is challenging for the researchers. This paper presents a new work for detecting forged handwriting words because width and amplitude of spectral distributions have the ability to exhibit unique properties for forged handwriting words compared to blurred, noisy and normal handwriting words. The proposed method studies spectral density and variation of input handwriting images through clustering of high and low frequency coefficients. The extracted features, which are invariant to rotation and scaling,are passed to a neural network classifier for the classification for forged handwriting words from other types of handwriting words (like blurred, noisy and normal handwriting words). Experimental results on our own dataset, which consists of four handwriting word classes, and two benchmark datasets, namely, caption and scene text classification and forged IMEI number dataset, show that the proposed method outperforms the existing methods in terms of classification rate.
\end{abstract}

Keywords:Handwriting recognition, Forgery detection, Fourier spectrum, Spectral distributions, Forged handwriting word detection.

\section{Introduction}

Due to rapid advancements in life and technology, crime rates are increasing exponentially in the world [1]. At the same time, techniques to find a solution to crime or forensic issues are also advancing in the research field. For example, the methods based on biometric features to protect properties from various crimes [2], are popular and successful in the market. However, sometimes, these systems are not reliable due to the creation of fake inputs and open environments [3]. Besides, the systems are computationally expensive. To overcome such limitations, systems use handwriting as 
a part of verification because handwriting can help us in identifying persons, persons' behaviors, gender orages, document verification, authentication, fraud document identification, etc [3]. In contrast to biometric feature based methods, handwriting process does not consume high processing time. Therefore, the number of applications and importance of handwriting increases. In the meantime, forging, tampering and creating fake writing also increases. Hence, detecting forged handwriting words is an unsolved issue for the researchers in the field of image processing and pattern recognition. The variations in handwriting make the problem more complex and challenging.

Forgery detection in images and videos, fraud document identification, printer identification and signature verification are all well-known topics in the literature [4]. There are several methods for addressing challenges of the above-mentioned topics. It is noted from the methods developed in the past that they work based on the fact that forgery operation introduces noises [5]. It is valid but noises can exist due to blurring, and flaws in devicesorwriting style in case of handwriting. Therefore, the methods developed in the past may not work for forged handwriting detection. Hence, we consider forged handwriting detection as a four-class classification problem in this work. The classification problem includes classes of Forged words created by copy-paste, insertion and deletion operations, Normal words (any word without forgery operation), Blurred words (we manually add Gaussian blur) and Noisy words (we add Gaussian noise to the words) as the sample images are shown in Fig. 1. Fig. 1 shows that it is hard to identify aforged word from the normal onewith our naked eyes. The proposed work aims to detect forged handwriting words irrespective of blur and noise.

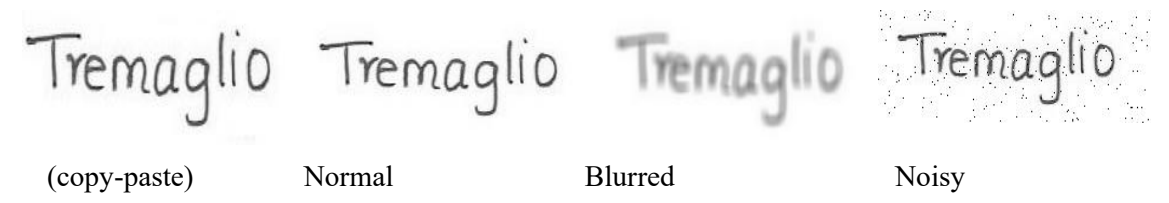

Fig. 1. Examples for four classes of handwriting words.

\section{Related Work}

As per our knowledge, there are not many methods for forged handwriting detection in literature. Therefore, we consider the methods that address forged caption text detection in video images, printer identification and fraud document identification as related work for reviewing.

In case of forged caption text detection in video images, caption text can beconsidered as tampered since it is edited. Shivakumara et al. [6] proposed caption text detection as a classification problem. The method works based on the combination of Canny and Sobel edge images along with ring radius transform. Xu et al. [7] proposed a method for detecting caption texts in videos by exploring Eigen values of input images. Roy et al. [8] proposed caption text detection by exploring DCT coefficients. In a similar way, Bhardwaj and Pankajakshan [9] explored tampered features using DCT coefficients in compression domain for detecting caption texts in video images. Re- 
cently, Roy et al. [10] proposed a method for caption text detection using video information. Shivakumara et al. [1] proposed a method for detecting forged IMEI (International Mobile Equipment Identity) based on RGB color channels and fusion concept.

It is noted from the above methods that the scope of the methods is to detect forgery in natural scene or video images. Since texts in these images appear as printed, the method may not be adequate to address the issue of handwriting texts in document images. There are methods for handwriting and printed texts in document images. Barbosa et al. [11] proposed text fraud detection in documents written with ballpoint pens. This method uses ink of ballpoint pens as features for fraud text identification. Elkasrawi and Shafait [12] proposed printer identification using supervised learning for document forgery detection. This approach explores prints based on noises produced by different printers for fraud document classification. Ahmed and Shafait [13] proposed forgery detection based on intrinsic document contents. This method explores similarity between blocks of an image to identify forged document identification. Khan et al. [14] proposed automatic ink mismatch detection for forensic document analysis. This method analyzes ink of different pens to find fraud documents. Luo et al. [15] proposed localized forgery detection in hyperspectral document images. This is an improved version of the above method, which explores ink quality in hyperspectral for fraud document identification. Bertrand et al. [16] proposed a system based on intrinsic features for fraudulent document detection. This approach extracts features or characters to match with the ground truth for fraud estimation. Based on mismatch scores, the method identifies fraud documents. Raghunandan et al. [17] proposed Fourier coefficients for fraud handwritten document classification through age analysis. This approach studies positive and negative coefficients for analyzing quality of images, which identifies it as old or new.Wang et al. [18] proposed a Fourier-residual method for fake printed text detection through printer identification. This method extracts features from residual given by Fourier transform for printer identification. Fahn et al. [3] proposed a text independent handwriting forgery detection system based on brachlet features and Gaussian mixture models. Cha et al. [19] proposed automatic detection of handwriting forgery. The method studies the contour of handwriting for forgery detection.

From the above discussion, it is observed that none of the methods considers blurred and noisy images for detecting forgery in images. This shows that the methods consider distortion, like noises created during forgery operation, as the main basis for achieving results. Therefore, the methods may not work well for images affected by forgery operation, blur and noises. Hence, this work explores new cues for detecting forged handwriting texts from images of blur and noises. The main intuition for the proposed method is the inconsistency caused in writing by forgery operation like creating fake characters using software, copy-paste, insertion and deletion compared to normal writing, blurred and noisy texts. This is because it is difficult to mimic dynamic aspects, such as speed and acceleration [19]. This results in wiggly, inconsistency and irregularity in writing. On the other hand, one can expect some regularity in case of blurriness and noisiness because blur degrades images with some consistency and noises do not affect contours of characters as they are isolated. Since 
noisesareisolated, they do not create problems for studying consistency and inconsistency in writing. In order to extract such unique observations, we introduce spectral densities and distributions. Motivated by the method [20] where it is stated that width and amplitude of spectrum directions have the ability to measure the quality of images affected by haze andblur, we explore the same spectral directions in a different way for classifying forged handwriting from blur, noise and clear images. The proposed work studies distribution of frequency coefficients and corresponding spectral densities for detecting forged handwriting words.

\section{Proposed Method}

As mentioned in the previous section, shape of spectral distribution is prominent for extracting distinct features to detect forged handwriting images. To study the shape of spectral distribution, the proposed method classifies high values of the spectrum into one cluster which is called Max cluster, and low values into another cluster which is called Min cluster. This is due to the relationship between high values which generally representedges (text), and low values which represent background. Since the considered problem is complex, studying the shape of the whole spectrum alone may not be adequate for achieving successful results. Therefore, the proposed method finds a unique relationship between the spectrums of text and background information. To make the features invariant to rotation and scaling, the proposed method estimates the principal axis for the binarized spectrum of Max and Min clusters. With respect to principal axis, the proposed method draws parallel and vertical lines over the Max and Min cluster spectrums. For the pixels of each parallel and vertical line of the spectrum of Max and Min clusters, the proposed method counts the number of white pixels and calculates the mean of intensity values as spectral density and spectral variations, respectively. To extract width and amplitude of the spectrum direction, the proposed method performs histogram operation for the number of white pixels and the mean intensity values of Max and Min cluster spectrums. The proposed method extracts statistical information from histogram distributions of the above operations. The extracted statistical features from histograms of a number of pixels and mean intensity values of Max and Min cluster spectrums are finally concatenated and fed to aNeural Network classifier for classification.

\subsection{Fourier Spectral Distributions}

For each input image, the proposed method obtains Fourier spectrum as defined in Equation (1)

$$
Y_{p+1, q+1}=\sum_{j=0}^{m-1} \sum_{k=0}^{n-1} X_{j+1, k+1} \omega_{n}^{k q} \omega_{m}^{j p}
$$

where, $Y$ denotes Fourier spectrum matrix forthe $m$-by- $n$ image matrix $X, \omega_{\mathrm{m}}$ and $\omega_{\mathrm{n}}$ are complex root of unity. The spectrums for four types of handwriting word images are shown in Fig. 2 (a), where the spectrum for each image appears different though the 
content is the same. This is a cue for us to explore the features for detecting forged handwriting words from blurred, noise and normal word images. The same cue is clearly visible in binary form of the spectrum of respective spectrums as shown in Fig. 2(b), where it is evident that the spectral distribution is unique for each type of word images. For obtaining the binary form of spectrum, we use well-known Otsu thresholding [21] as it is good for separating foreground and background for plain document images.
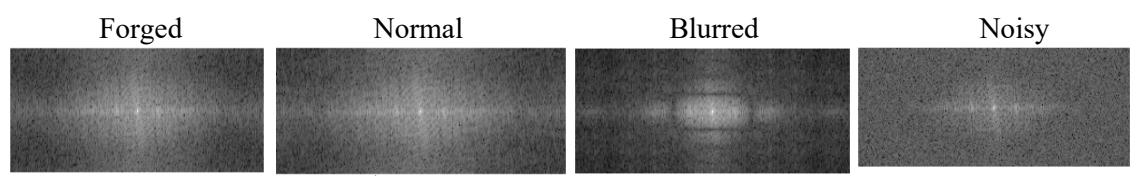

(a) Fourier spectrum for the four types of images
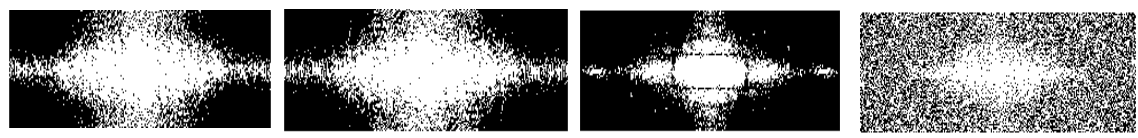

(b). Binary form of Fourier spectrum for the four types of images

Fig. 2. Fourier spectrum distribution for classification.

\subsection{Spectral Density and Variance based Features for Forged Handwriting Classification}

For the frequency coefficient values of corresponding white pixels in the spectrum, the proposed method performs K-means clustering for classifying high frequency coefficients into Max and low frequency coefficients into Min cluster. The spectrum in binary form is obtained for the Max and Min cluster frequency coefficients as respectively shown in Fig. 3(a) and Fig. 3(b) for all the four types. It is noted from Fig. 3(a) and Fig. 3(b) that shapesof spectrum differ from one image to another. This observation motivated us to study the shape of the spectrum of Max and Min clusters. For this target, the proposed method estimates the principal axis and draws parallel and vertical lines with respect to principal axis as shown in Fig. 3(a) and Fig. 3(c) for parallel lines and Fig. 3(b) and Fig.3(d) for vertical lines. For pixels of parallel and vertical lines of Max and Min clusters, the proposed method counts the number of pixels as spectral density features and calculates the mean of intensity values as spectral variation features as defined in Equation (2). As pointed out in the Proposed Method Section, width and amplitude of spectrum are good for achieving better results, the proposed method thus performs histogram operation for spectral density and spectral variation values vs. row and column for respective Max and Min clusters. Further, the proposed method finds the average peak in histograms and considers it as a reference as defined in Equation (3). The deviations from the reference to the lowest peak either on side of the reference line are calculated as defined in Equation (4) and Equation (5). This results in two features as defined in Equation (6) and Equation (7) for histogram of parallel lines of Max clusters with respect to spectral density features, 
and two more features for histogram of vertical lines of Max cluster of spectral density features. For histograms of Min clusters with respect to spectral density features, the proposed method obtains four features. In total, for the spectral density features, we extract 8 features.

Similarly, for spectral variation based features, the proposed method calculates deviation as a spectral density feature. In addition to these features, the proposed method considers value reference line as an additional feature as it represents spatial variations in images. As a result, for spectral variation based features, the proposed method extracts 12 features, of which 6 are from histogram of parallel and vertical lines of Max cluster, and the other 6are from parallel and vertical lines of Min cluster. In total, the proposed method extracts 20 features from input images. The process of extracting features is illustrated in Fig. 4(a), where the average peak and the lowest peak either side of the reference lines are shown in dark color for both the histograms of spectral density (left one) and variation (right one) based features. The deviation from the reference lines is also illustrated in Fig. 4(a). Line graphs for the feature vector containing 20 features of four types of input images are plotted in Fig. 4(b), where it is observed that the line graphs of respective feature vectors of the four types behave differently. This shows that the extracted features have a discriminative power for classifying forged handwriting word from blurred, noise and normal words images.

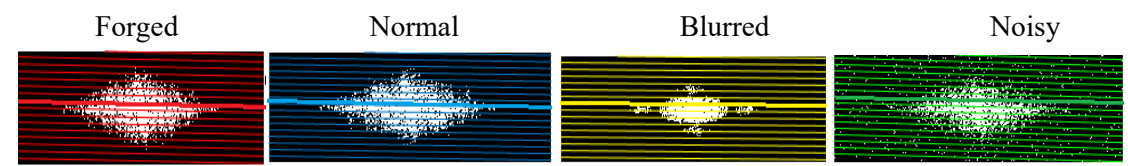

(a) Parallel lines with respect to principal axis for max cluster results.
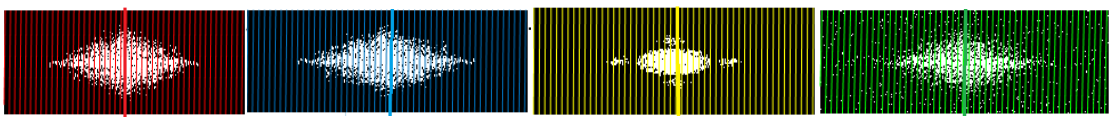

(b) Vertical lines with respect to principal axis for max cluster results.
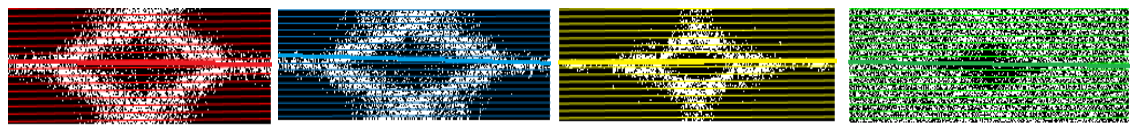

(a) Parallel lines with respect to principal axis for min cluster results.
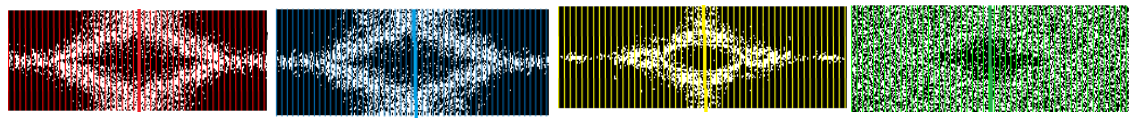

(b) Vertical lines with respect to principal axis for min cluster results

Fig. 3. Parallel and vertical lines with respect to principal axis for the spectrum of max and min cluster.

$$
A V=\frac{\sum_{i=1}^{N w p} n_{i}}{N w p}
$$


where, $N w p$ is the number of white pixels on that particular line, and $n_{i}$ is the intensity of that pixel of the Fourier spectrum.

$$
\begin{gathered}
x_{\text {Nmean }}=x \text { value of }\left(\min _{\mathrm{y} \in N}\left(\mathrm{y}-\operatorname{avg}\left(N_{\text {Max }}^{\text {parallel }}\right)\right)\right) \\
x_{N 1}=x \text { value of }\left(\min _{0 \leq k<\mathrm{x}_{\text {Nmean }}}\left(y_{k}\right)\right) \\
x_{N 2}=x \text { value of }\left(\min _{\mathrm{x}_{\mathrm{Nmean}}<k \leq \mathrm{m}}\left(y_{k}\right)\right) \\
\text { feature }_{1}=x_{N \text { mean }}-x_{N 1} \\
\text { feature }_{2}=x_{N 2}-x_{N \text { mean }}
\end{gathered}
$$

where, $x$ (the value of spectral density/spectral variation) denotes the values of $\mathrm{X}$ axis, $y$ denotes (the number of white pixels for each $x$ ) the value on $\mathrm{Y}$ axis, $N_{\text {Max }}^{\text {paralle }}$ denotes the number of white pixels in the plot of parallel lines of Max cluster, $x_{\text {Nmean }}$ denotes the nearest value of $x$ at which the average of $N_{\text {Max }}^{\text {parallel }}$ is obtained, $x_{N 1}$ and $x_{N 2}$ denote deviation of minimum values on either side of the reference line.
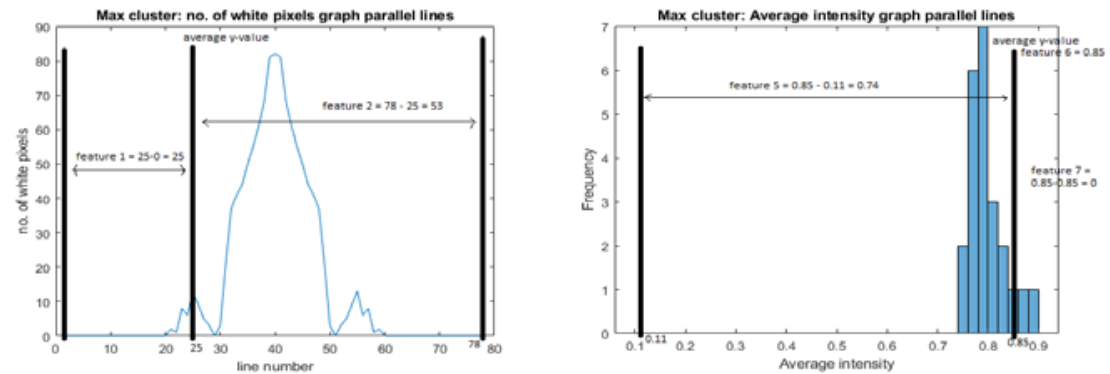

(a) Illustration for extracting features from spectral density and variations histograms.

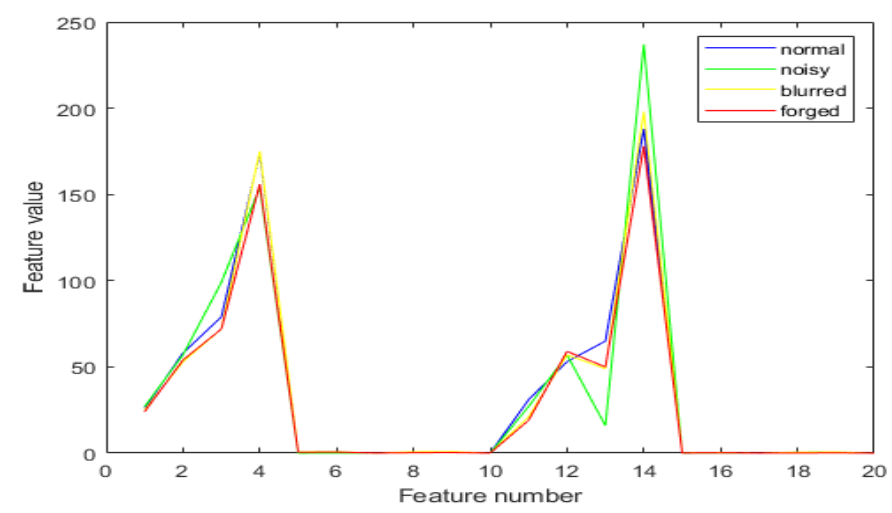

(b) The extracted 20 features distribution of four types of images.

Fig. 4. Visual clues for classification of forged handwriting word from blurred, noisy and normal word images. 
For classification, we propose to use a neural network classifier in this work because as discussed the previous section, the features are good enough to distinguish forged handwriting words from the other images. Therefore, we prefer to use a simple classifier. The proposed method uses a feed-forward neural network with 20 hidden layers. For determining weights and updating, the proposed method uses the Levenberg-Marquardt back-propagation procedure to update weights and bias terms of the model. The error is calculated using the mean squared error and the process of training stops when the maximum number of epochs isreached or the performance is minimized to the goal. For experiments, we divide the dataset into $80 \%$ of samples for training, $10 \%$ for testing and $10 \%$ for validation. The process of training and the structure of neural networks areshown in Fig. 5,where we can see how the proposed work trains parameter values for classification experiments.

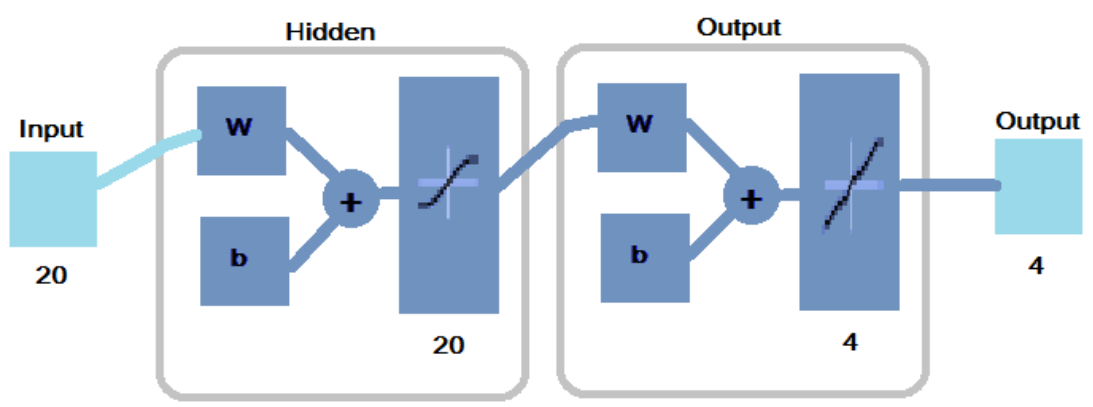

Fig. 5. Structure of the training neural network classifier for classification Forged

\section{$4 \quad$ Experimental Results}

For evaluating the performance of the proposed method, since this is the first work for forged handwriting word detection, there are no standard datasets available in literature. We thus create our own dataset, which includes 200 images for each class and hence the size of our dataset is 800 images. For creating forged words, we use standard operations, namely, copy-paste which copies words/characters from one source to a target place, deletion which erases a few characters in the words, and insertion which adds character words to the original text. Sample images for each operation are shown in Fig. 6(a)-Fig. 6(c), respectively, where one can see the effect of forgery operation for each case. If we compare forged words with normal words, it is hard to notice the change in content without any knowledge of dataset and problem. This ensures that the created dataset is good enough for evaluating the performance of the proposed forged handwriting word detection. To make sure that the proposed method does not consider the basis which the existing methods consider for forgery detection, we created two more classes of blurred and noise images as shown in Fig. 6(d) and Fig.6(e), respectively. For creating blurred and noisy images, we use Gaussian 
functions for adding blur and pepper-salt noise function for adding noises at some different levels. Therefore, the considered problem is a four-class classification problem instead of two-class classification.

In order to test the objectiveness of the proposed method, we also consider two standard datasets, namely, forged caption text detection [8] in video images which consists of 1075 images, and forged IMEI number detection [1] which consists of 1000 images. In total, 2875 images are considered for experimentation in this work. In case of caption and scene text dataset, caption text images are considered as forged images as they are edited, and scene text images are considered as normal ones as these are part of images. In case of forged IMEI number dataset, images of forged IMEI numbers are one class, and the original images are considered as another class. Although these data are not handwritten, the reason to choose these two datasets as the standard datasets for experimentation is show how our work behaves on printed text. Also these datasets are available and use the same operations for creating forged images, which is another reason. Experiments on these datasets show that the proposed method is robust and can be used for texts in video images and printed numerals.

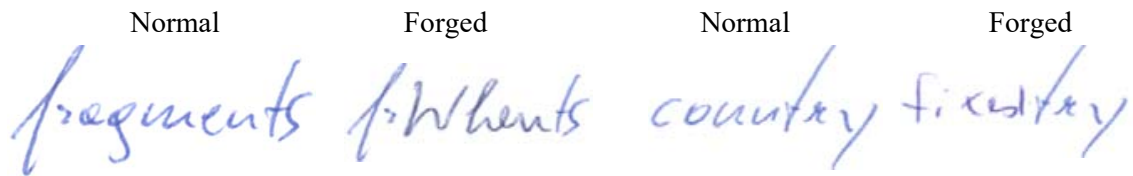

(a) Copy-paste operation for creating forged words.

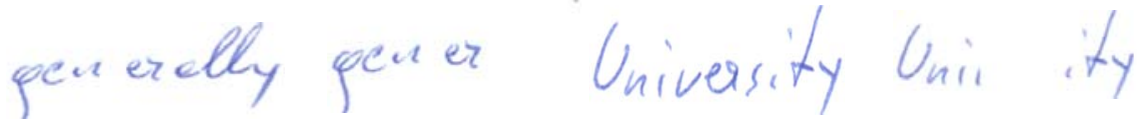

(b) Deletion for creating forged words

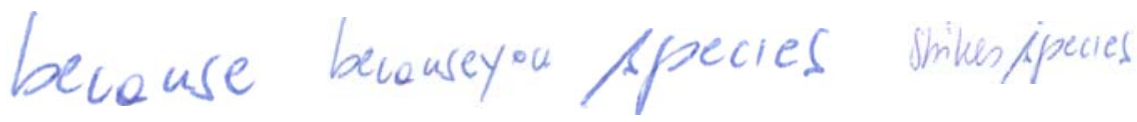

(c) Insertion for creating forged words

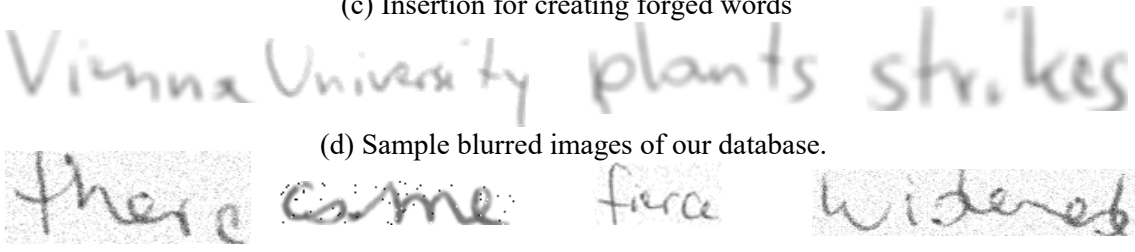

(e) Examples for noisy images.

Fig. 6. Sample images for forged, blurred and noisy handwriting word images

To show the usefulness of the proposed method, we implement three existing methods to compare with the results of the proposed method. Wang et al. [18] proposed Fourier-residual for printer identification, which extracts statistical, spatial and texture features for residual images given by Fourier reconstructed images. Elkasrawi and Shafait[12] proposed printer identification using supervised learning for document forgery detection, and they use residual in a different way. Bhardwaj et al. [9] proposed a method for forged caption text detection in video images. The reason to choose the first two methods is that the objective of these methods is forgery detection in document images including handwriting also, which is the same as the proposed 
method. The third one is to show that the methods, which are developed for forged caption text detection in video, may not be suitable for forged handwriting word detection. In addition, the method which uses distortion created by forgery operation as the main basis for forgery detection, may not work well for forged handwriting word detection from blurred, and noisy word images. Note that since the methods $[12,18]$ used classifiers for forgery detection in images, we can extend the same classifier for the proposed four classes. Therefore, we run these two methods on our four class dataset as well as the two class dataset of our dataset and the standard dataset. However, the method [9] uses threshold for two class classification because it is developed for two class problem. Hence, we run this method on two classes of our dataset and the standard dataset for comparative study in this work but not for three classes. For measuring the performances of the proposed and existing methods, we use standard measures, namely, confusion matrix and classification rate as defined in Equation (8), which is the mean of all the diagonal elements of the confusion matrix. For all the above three datasets, since there is no ground truth, we count manually for calculating measures in this work.

$$
C R=\frac{T P}{N_{\text {total }}} \times 100
$$

where, $T P$ are the total images labeled correctly and $N_{\text {total }}$ are the total number of test images.

\subsection{Experiments on Forged Handwriting Word Detection}

Quantitative results of the proposed and existing methods for four-class classification are reported in Table 1, where it is noted that the proposed method is better than two existing methods in terms of Classification Rate (CR). When we compare the two existing methods, Wang et al. score the better results compared to Elkasrawi and Shafait. This is due to the lack of discriminative power in feature extraction in case of Elkasrawi and Shafait's method. At the same time, Wang et al. take advantage of statistical, texture and spatial features for forged document identification compared to Elkasrawi and Shafait's method. However, the extracted features may not be robust to cope with the challenges posed by different writing styles and variations compared to printed texts in document images, and hence the existing method [18] reports poor results for forged handwriting word detection from blurred and noise words. In addition, the existing methods are developed based on the fact that noises created by different operations or sources exhibit different natures. This is not necessarily true for the proposed problem, which includes noises created by blur and different noise levels. The same thing is true for the other existing method [12]. In order to test the ability of the proposed method for two classes, we conduct experiments on two classes, namely, Forged vs Normal, Forged vs Blurred and Forged vs Noise as reported in Table 2. Table 2 shows that the proposed method is the best at classification rate compared to the existing methods for all the three combinations. It is observed from Table 2 that Bhardwaj and Pankajakshan [9] fail to achieve better results compared to the other existing methods and the proposed method for all three combinations. The main reason is the fixing of a constant threshold because afixed threshold may not be optimal for all different combinations and datasets. It is expected that the proposed and the existing methods score better results for two classes compared to four classes reported in Table 1. Interestingly, it is noted from Table 2 that for Forged vs Noise classes, Wang et al. 
and the proposed method achieve $100 \%$ classification rate. It is evident that these two methods have the ability to differentiate between the distortions created by noises and forgery operation, while the other methods do not.

Table 1. Classification rates of the proposed and existing methods on forged handwriting dataset for four classes (in \%).

\begin{tabular}{|c|c|c|c|c|c|c|c|c|c|c|c|c|}
\hline Methods & \multicolumn{4}{|c|}{ Proposed method } & \multicolumn{4}{c|}{ Wang et al. [18] } & \multicolumn{3}{c|}{ Elkasrawi and Shafait [12] } \\
\hline Classes & Forged & Normal & Blur & Noise & Forged & Normal & Blur & Noise & Forged & Normal & Blur & Noise \\
\hline Forged & 85.7 & 4.8 & 9.5 & 0.0 & 71.4 & 25 & 1.8 & 1.8 & 81.8 & 3.6 & 5.5 & 9.1 \\
\hline Normal & 20.0 & 70.0 & 10.0 & 0.0 & 25 & 57.8 & 7.8 & 9.4 & 0 & 58.2 & 5.5 & 36.3 \\
\hline Blurred & 15.8 & 15.8 & 63.2 & 5.2 & 1.8 & 9 & 78.2 & 11 & 4.8 & 9.5 & 60.3 & 25.4 \\
\hline Noisy & 0.0 & 0.0 & 10.0 & 90.0 & 8 & 14.3 & 1.5 & 76.2 & 3.25 & 14 & 26.5 & 56.25 \\
\hline CR & \multicolumn{4}{|c|}{77.5} & & \multicolumn{4}{c|}{70.1} & & \multicolumn{5}{c|}{64.14} \\
\hline
\end{tabular}

Table 2. Classification rates of the proposed and existing methods on forged handwriting dataset for two classes (in \%).

\begin{tabular}{|c|c|c|c|c|c|c|c|c|}
\hline Methods & \multicolumn{2}{|c|}{ Proposed } & \multicolumn{2}{|c|}{ Bhardwaj et al [9] } & \multicolumn{2}{|c|}{ Wang et al [18] } & \multicolumn{2}{|c|}{$\begin{array}{c}\text { Elkasrawi and } \\
\text { Shafait [12] }\end{array}$} \\
\hline Classes & Forged & Normal & Forged & Normal & Forged & Normal & Forged & Normal \\
\hline Forged & 72.2 & 27.8 & 66.0 & 34.0 & 90.6 & 9.4 & 70.3 & 29.7 \\
\hline Normal & 18.2 & 81.8 & 72.0 & 28.0 & 20 & 80 & 25.5 & 74.5 \\
\hline $\mathrm{CR}$ & \multicolumn{2}{|c|}{77.5} & \multicolumn{2}{|c|}{49.7} & \multicolumn{2}{|c|}{$\frac{1}{85.3}$} & \multicolumn{2}{|c|}{$\frac{1}{72.4}$} \\
\hline Classes & Forged & Blur & Forged & Blur & Forged & Blur & Forged & Blur \\
\hline Forged & 95.8 & 4.2 & 66.0 & 34.0 & 94 & 6 & 96.4 & 3.6 \\
\hline Blur & 0.0 & 100 & 0.0 & 100.0 & 22.82 & 77.18 & 6.25 & 93.75 \\
\hline $\mathrm{CR}$ & \multicolumn{2}{|c|}{97.5} & \multicolumn{2}{|c|}{83} & \multicolumn{2}{|c|}{87.6} & \multicolumn{2}{|c|}{95} \\
\hline Classes & Forged & Noise & Forged & Noise & Forged & Noise & Forged & Noise \\
\hline Forged & 100 & 0.0 & 66.0 & 34.0 & 100 & 0.0 & 70.3 & 29.7 \\
\hline Noise & 0.0 & 100.0 & 95.0 & 5.0 & 0.0 & 100 & 23.6 & 76.4 \\
\hline $\mathrm{CR}$ & \multicolumn{2}{|c|}{100} & \multicolumn{2}{|c|}{35.50} & \multicolumn{2}{|c|}{100.0} & \multicolumn{2}{|c|}{73.35} \\
\hline
\end{tabular}

\subsection{Experiments on Forged Caption Text Detection in Video Images}

As mentioned in Experimental Section, to test the proposed method on different dataset, we conduct experiments on forged caption text detection from scene texts in video images. Sample successful classification images for caption and scene texts in videos can be seen in Fig. 6(a), where it can be seen that the distortion created by editing over video is not visible in case of caption texts, while we can see blur and degradations created by perspective distortion in case of scene texts. As a result, this dataset is challenging as the proposed dataset. Quantitative results reported in Table 3 for the proposed and existing methods show that the proposed method is better than the existing methods in terms of classification rate. It is noted from Table 3 that though the method proposed by Bhardwaj and Pankajakshan[9] is developed for forged caption text detection in video, it fails to achieve the best results. This shows that fixing threshold for all the three different datasets is not easy. Therefore, one cannot expect consistent results from the Bhardwaj and Pankajakshan's [9] method. However, the other two existing methods report reasonable results compared to the proposed method. 
Overall, one can argue that the proposed method is robust to different datasets and different numbers of classes.

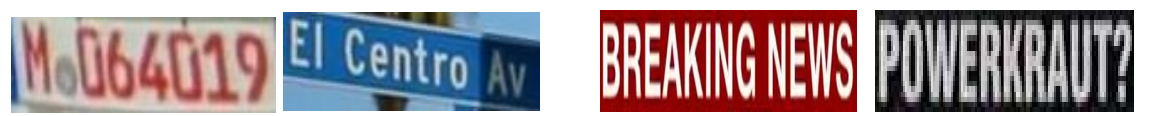

(a) Examples for scene and caption (forged) text of Roy et al. [8] dataset.
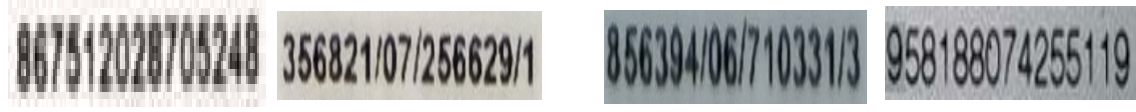

(b) Examples for normal and forged words IMEI number of Shivakumara et al da-

Fig. 6. Successful classification of the proposed method on scene-caption and IMEI number images

Table 3. Classification rates of the proposed and existing methods on scene text dataset for two classes (in \%).

\begin{tabular}{|c|c|c|c|c|c|c|c|c|}
\hline \multirow{2}{*}{ Methods } & \multicolumn{2}{|c|}{ Proposed } & \multicolumn{2}{c|}{ Bhardwaj et al. [9] } & \multicolumn{2}{c|}{ Wang et al. [18] } & \multicolumn{2}{c|}{$\begin{array}{c}\text { Elkasrawi and } \\
\text { Shafait [12] }\end{array}$} \\
\hline Classes & Scene & Caption & Scene & Caption & Scene & Caption & Scene & Caption \\
\hline Scene & 100 & 0 & 46.7 & 53.33 & 93.3 & 6.7 & 87.6 & 12.4 \\
\hline Caption & 3.5 & 96.5 & 72.8 & 27.2 & 3.6 & 96.4 & 15.3 & 84.17 \\
\hline CR & \multicolumn{2}{|c|}{98.1} & \multicolumn{2}{c|}{36.9} & \multicolumn{2}{c|}{94} & \multicolumn{2}{c|}{86.15} \\
\hline
\end{tabular}

\subsection{Experiments on Forged IMEI Number Detection}

Sample successful results of the proposed method are shown in Fig. 6(b), where it is noted that there is no visible evidence to differentiate forged IMEI number from the normal IMEI number. As a result, the distortion created by forgery operation may overlap with the distortions in normal images because the scope is limited to only numerals. In addition, the dataset includes images of forging only a few numerals but not the whole of images unlike forged caption text detection and forged handwriting word detection. Therefore, this dataset is complex compared to the other two datasets. It is confirmed from the results reported in Table 4 that the proposed and existing methods score poor results compared to the other two datasets in spite of having two classes. This is due to overfitting problem. However, Elkasrawi and Shafait [12] score the best for this dataset compared to the proposed and the other existing methods. This shows that the method [12] is not consistent for different datasets when we compare the performances of the proposed and Wang et al.'s methods. Overall, though the proposed method is developed for forged handwritten word detection from blurred and noisy words, it achieves the best result for almost all the experiments of different datasets. Therefore, we can conclude that the proposed method is robust, generic and can be extended to more classes and more images for different applications with little modification. This is because the proposed features are extracted based on spectrum direction and distribution, which do not depend on specific content of images.

Table 4. Classification rates of the proposed and existing methods on forged IMEI number dataset for two classes (in \%). 


\begin{tabular}{|c|c|c|c|c|c|c|c|c|}
\hline Methods & \multicolumn{2}{|c|}{ Proposed } & \multicolumn{2}{|c|}{ Bhardwaj et al. [9] } & \multicolumn{2}{|c|}{ Wang et al. [18] } & \multicolumn{2}{|c|}{$\begin{array}{c}\text { Elkasrawi and } \\
\text { Shafait [12] }\end{array}$} \\
\hline Classes & Original & Forged & Original & Forged & Original & Forged & Original & Forged \\
\hline Original & 57.8 & 42.2 & 10.2 & 89.8 & 47.8 & 52.1 & 45.2 & 54.8 \\
\hline Forged & 41.8 & 58.2 & 5.8 & 94.2 & 41.0 & 59.0 & 27.0 & 73 \\
\hline $\mathrm{CR}$ & \multicolumn{2}{|c|}{58.0} & \multicolumn{2}{|c|}{51.5} & \multicolumn{2}{|c|}{53.5} & \multicolumn{2}{|c|}{59.1} \\
\hline
\end{tabular}

Sometimes, due to the large variations in writing style, there is a chance of misclassification as the shown sample misclassified images in Fig. 7(a) and Fig. 7(b). This is valid because the proposed features are extracted based on the global information, which may not be robust when images do not contain enough characters. Therefore, there is a scope for improvement and the method can be extended to overcome this limitation by exploring the combination of local and global information.

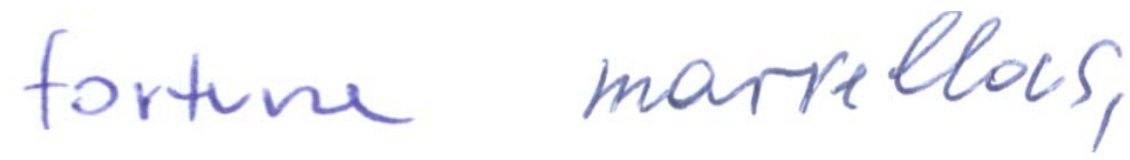

(a) Normal words are misclassified as forged words

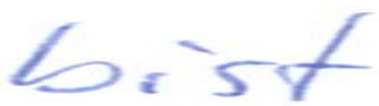

(b) Normal words misclassified as blurred words

Fig. 7. Unsuccessful classification of the proposed method for some cases.

\section{$5 \quad$ Conclusion and Future Work}

In this work, we have proposed a new method for forged handwriting word detection from blurred, noisy and normal (clear) words. The proposed method explores spectral density and variations for extracting features based on the fact that width and amplitude of spectrum direction are sensitive to distortion created by forged operation, blur and noises. To extract such an observation, the proposed method divides high and low frequency coefficients to study the unique relationship between text and background information in input images. The extracted features are fed to a neural network classifier for classification. To evaluate the proposed method, we have tested the proposed method on forged handwriting, forged caption text and forged IMEI number datasets. Experimental results on different datasets show that the proposed method is robust to different datasets and different number of classes compared to the existing methods. As pointed out in the experimental section, there is a scope for the improvement and extension to overcome the limitations by exploring combination of local and global information in the future. 


\section{References}

1. P. Shivakumara, V. Basavaraja, H. S. Gowda, D. S. Guru, U. Paland T. Lu,"A New RGB Based Fusion for Forged IMEI Number Detection in Mobile Images”, In Proc. ICFHR, 2018, pp 386-391.

2. M. A. Ferrer, A. Morales, J. F. Vargas, I. Lemos and M. Quintero, "Is it possible to automatically identify who has forged my signature? Approaching to the identification of static signature forger", In Proc. DAS, 2012, pp 175-179.

3. C. S. Fahn, C. P. Lee and H. I. Chen, "A text independent handwriting forgery detection system based on branchlet features and Gaussian mixture models", In Proc. PST, 2016.

4. T. M. Ghanim and A. M. Nabil, "Offline signature verification and forgery detection approach", In Proc. ICCES, 2018, pp 293-298.

5. R. Kumar, N. R. Pal, B. Chanda and J. D. Sharma, "Forensic detection of fraudulent alterations in ball point pen strokes", IEEE Trans. IFS, Vol. 7, No. 2, pp 809-820, 2012.

6. P. Shivakumara, N. V. Kumar, D. S. Guru and C. L Tan, "Separation of graphics (superimposed) and scene text in videos", In Proc. DAS, pp 344-348, 2014.

7. J. Xu, P. Shivakumara, T. Lu, T. Q. Phan and C. L. Tan, "Graphics and Scene Text Classification in Video", In Proc. ICPR, pp 4714-4719, 2014.

8. S. Roy, P. Shivakumara, U. Pal, T. Lu and C. L. Tan, "New Tampered Features for Scene and Caption Text Classification in Video Frame", In Proc. ICFHR, pp 36-41, 2016.

9. D. Bhardwaj, V. Pankajakshan, "Image Overlay Text Detection Based on JPEG Truncation Error Analysis", IEEE Signal Process. Lett. pp 1027-1031, 2016.

10. S. Roy, P. Shivakumara, U. Pal, T. Lu and A. W. B. A. Wahab, "Temporal integration of word-wise caption and scene text identification”, In Proc. ICDAR, pp 350-355, 2017.

11. R. D. S. Barboza, R. D. Lins, E. D. F. D. Lira and A. C. A. Camara, "Later added strokes of text fraud detection in documents written with ballpoint pens", In Proc. ICFHR, pp 517-522, 2014.

12. S. Elkasrawi and F. Shafait, "Printer identification using supervisedlearning for document forgery detection", In Proc. DAS, 146-150, 2014.

13. A. Ahmed and F. Shafait, "Forgery detection based on intrinsic document features", In Proc. DAS, 252-256, 2014.

14. Khan, F. Shafait and A. Mian, "Automatic Ink mismatch detection for forensic document analysis", Pattern Recognition, Vol. 48, pp 3615-3626, 2015.

15. Z. Luo, F. Shafait and A. Mian, "Localized forgery detection in hyperspectral document images", In Proc. ICDAR, 496-500, 2015.

16. R. Bertrand, P. G. Kramer, O. R. Terrades, P. Franco and J. M. Ogier, "A system based on intrinsic features for fraudulent document detection”, In Proc. ICDAR, pp 106-110, 2013.

17. K. S. Raghunandan, P. Shivakumara, B. J. Navya, G. Pooja, Navya, N. Prakash, G. H. Kumar. U. Pal and T. Lu, "Fourier coefficients for fraud handwritten document classification through age analysis", In Proc. ICFHR, pp 25-30, 2016.

18. Z. Wang, P. Shivakumara, T. Lu, M. Basavanna, U. Pal and M. Blumenstein, "Fourierresidual for printer identification", In Proc. ICDAR, pp 1114-1119, 2017.

19. S. H. Cha and C. C. Tappert, "Automatic detection of handwriting forgery", In Proc. IWFHR, 2012.

20. R. Wang, W. Li, R. Li and L. Zhang, "Automatic blur type classification via ensemble SVM", Signal Processing: Image Communication, 71, 2019, pp 24-35.

21. N. Otsu, "A Threshold Selection Method from Gray-Level Histograms." IEEE Trans. Man, and Cybernetics. Vol. 9, No. 1, 1979, pp. 62-66. 